\title{
TPP1 Regulates hTERT Expression and Predicts Early Malignant Event and Prognosis of Cervical Cancer
}

\section{Qiaoli Wang}

Wuhan University Second Clinical Hospital: Wuhan University Zhongnan Hospital

\section{Caifeng Gong}

Chinese Academy of Medical Sciences Cancer Institute and Hospital: Cancer Hospital Chinese Academy of Medical Sciences https://orcid.org/0000-0002-1864-8206

\section{Hui Yang}

Wuhan University Second Clinical College: Wuhan University Zhongnan Hospital

\section{Fuxiang Zhou}

Wuhan University Second Clinical College: Wuhan University Zhongnan Hospital

\section{Qiuji Wu ( $\nabla$ wuqiuji@126.com )}

Zhongnan Hospital of Wuhan University https://orcid.org/0000-0002-4655-3108

\section{Yunfeng Zhou}

Wuhan University Second Clinical Hospital: Wuhan University Zhongnan Hospital

\section{Research}

Keywords: cervical cancer, telomere-binding protein 1, human telomerase reverse transcriptase, prognosis, biomarker

Posted Date: August 17th, 2021

DOI: https://doi.org/10.21203/rs.3.rs-794147/v1

License: (c) (i) This work is licensed under a Creative Commons Attribution 4.0 International License. Read Full License 


\section{Abstract}

Background: Cervical cancer is one of the most common deadly cancer in women worldwide. However, identifying specific biomarkers is still needed. Telomere-binding protein 1 (TPP1) is vital to telomerase activity. However, the role of TPP1 in cervical cancer and its association with human telomerase reverse transcriptase (hTERT) is unclear.This study aimed at exploring the role of telomere-binding protein 1 (TPP1) in cervical cancer development and progression, and potential mechanisms.

Methods: Tissue samples from a total of 274 participants were enrolled for the evaluation of protein expression, 156 of whom diagnosed withcervical cancers, 102 with cervical intraepithelial neoplasia (CIN) and 16 with normal cervix. In addition, in vitro cellular models with cervical cancer cell lines Hela, Siha, and C33a were transfected by TPP1-siRNAand protein expression of TPP1 and hTERT were assessed.

Results: Compared with normal cervix, TPP1 expression was significantly higher in CIN-III and cervical cancers $\left(P<0.001\right.$ for both). High expression of TPP1alone $\left(P_{\text {log-rank }}=0.047\right)$ andhigh co-expression of TPP1/hTERT ( $\left.P_{\text {log-rank }}=0.005\right)$ weresignificantly associated with worse survival of cervical cancer patients.After adjusting for well-known prognosis factors, hazard ratio was 2.03 (95\% confidence interval [Cl] 0.99-4.16)for high expression of TPP1 and 2.01(95\% Cl 1.10-3.67) for high co-expression of TPP1/hTERT. TPP1 and hTERT expressions were positively correlated atall levels of cervical lesions $(r=0.524, P<0.001)$. Knockdown of TPP1 decreased hTERT mRNA and protein expression.

Conclusions: High expression of TPP1 might be an early event during cervical cancer development and could be served as apotential prognosis biomarker, especially when used together with hTERT. TPP1 might regulate hTERT expression with detailed underlying mechanisms warrant further investigation.

\section{Introduction}

In 2018, the Global Cancer Observatory (GLOBOCAN) estimated 569,847 new cervical cancer cases and 311,365 cancer-related deaths, making it the fourth most common cancer and the fourth most deadly cancer in women worldwide [1]. Cervical cancer severely affects women's health with more young women aged 15 to 49 years diagnosed during the last decade [2]. To detect precancerous lesions, to screen early cancers, and to improve patient survival, novel biomarkers and therapeutic targets are urgently needed.

Telomere, a DNA-protein complex locating at the end of chromosomes, together with telomere-binding proteins preserves chromosomal integrity and regulating cell cycles. Maintenance of telomere length and function is crucial during carcinogenesis, which is usually regulated by both telomerase and telomerebinding proteins [3]. Telomere-binding protein 1 (TPP1, also known as TINT1 or PTOP or PIP1) is a vital component of the telomere-binding proteins shelterin complex [4]. TPP1 binds to telomerase and protects chromosome ends from cellular DNA damages. TPP1 also initiates the functions of DNA repair machineries by recruiting telomerase to telomeres. These functions stimulate telomerase activity and promote telomere elongation in telomerase-positive cells [5-7]. Additionally, TPP1 combines with another shelterin complex component named protection of telomeres 1 (POT1) to form a stable heterodimer, 
which maintains genome stability and regulates telomerase-mediated telomere extension [6, 8-10]. Human telomerase reverse transcriptase (hTERT) is a fundamental element of telomerase and is known to be associated with cell stemness, cell proliferation and resistance to chemotherapy and radiotherapy in various cancers [11-14]. Similar to the finding that inhibition of hTERT activity shortens the telomere length and hinders its functions, TPP1 silent mutation using the CRISPR-Cas9 technology is also sufficient to shorten telomeres in human cells [15]. It is suspected that TPP1 may regulate telomere length partly through hTERT. hTERT was shown to be a good biomarker for identifying and treating several cancers, but it has limited effect on cervical cancer [16-18]. Identifying specific biomarkers for cervical cancer is therefore needed. This study aims to clarify the expression and clinical value of TPP1 and its association with hTERT in cervical cancer development and prognosis.

\section{Patients And Methods}

Study design

A total of 274 cervical tissue samples were collected in this study. All eligible cervical cancer patients $(n=$ 156) were identified in Zhongnan Hospital of Wuhan University (Wuhan, China) between January 1, 2003, and December 31, 2008. Another 102 cervical intraepithelial neoplasia (CIN) tissues and 16 normal cervical tissue samples were collected from Fanpu Biotech Inc. (Guilin, Guangxi, China) during the same study period. All cervical cancer patients and CINs patients received either cervical biopsy or surgical resection, and normal cervical tissues were collected from participants who had hysteromyoma resection but without other cervical diseases. Inclusion criteria of cancer patients were aged $>18$, diagnosed with cervical cancer as primary cancer during the study period, received standardized treatments, and had complete medical records. Patients were followed up through outpatient visits in the hospital and a regular phone call by the Clinical Follow-up Unit. Follow-up started from the date of cancer diagnosis to death from any cause, lost to follow up, or end of the study (January 31, 2014), whichever came first.

Clinical characteristics of cervical cancer cases were collected through medical records, which included age, tumor diameter, the International Federation of Gynecology and Obstetrics (FIGO) stage, differentiation, lymphatic invasion, vagina invasion, parauterus invasion, and pathological subtype. Overall survival (OS) time was defined as the date of surgery to the date of death from any cause, or the last date of follow-up, whichever came first.

Immunohistochemical staining

Hematoxylin and eosin stain were used to confirm the primary pathological diagnosis. Immunohistochemistry (IHC) test was performed to assess TPP1 and hTERT expression (Fig. 1A and Fig. S1A) following manufacturer's instructions. Primary antibodies against TPP1 (Abcam, ab57595, dilution of 1:100) and hTERT (Abcam, ab183105, dilution of 1:30) were incubated on $4 \mu \mathrm{m}$-section samples for 1.5 hours at room temperature. Two pathologists blinded to the outcome independently assessed the protein expression using a predefined rating system. We used IHC rating scales for hTERT according to the previously published method [19], the total score of hTERT was rated by a combination of intensity 
and percentage of positive cells (Table S1). TPP1 was nuclear expressed and almost all the positive cells were strongly expressed. Therefore, TPP1 score was defined according to the percentage of positive cells: $<25 \%$ of positive cells as negative $(-), 10-49 \%$ as weakly positive $(+), 50-74 \%$ as moderately positive $(++)$, and $\geq 75 \%$ as strongly positive (+++). For each cervical tissue sample, two blocks per case were evaluated and an averaged score was applied. A discrepancy of scores from two pathologists was solved by re-evaluating and rescoring the samples together. For better interpretations, we defined both negative $(-)$ and weakly positive $(+)$ rates as low expression, and moderately positive (++) and strongly positive $(+++)$ rates as high expression.

Cell transfection

Three human cervical cancer cell lines, Hela, C33a, and Siha were purchased from the Institute of Life Sciences, Chinese Academy of Science (Shanghai, China). Cells were cultured in DMEM (Gibco, Life Technologies Corporation, UK) supplemented with $10 \%$ fetal bovine serum (FBS) and $100 \mathrm{UI} / \mathrm{mL}$ penicillin/streptomycin and dissociated by Accumax (Gibco). The plasmids (purchased from GenePharma, Shanghai) was used to knock down TPP1 with TPP1-siRNA sequence of 5'GTGGTACCAGCATAGCCT-3' by means of Lipofectamine (LipofectamineTM2000, Invitrogen, US). Cells were plated in two six-well plate at $2 \times 10^{5}$ cells per well. After 24 hours of incubation, $5 \mu$ l of transfection reagent Turbfect (Thermo Fisher Scientific, USA) was mixed with $8 \mu$ l of siRNA in 500 $\mu$ DMEM and then the mixture was added to the cell culture medium. Cells were collected after 48 hours from the transfection to perform transfection validation, RNA and protein extraction. Transfection efficiency was presented as the intensity and the percentage of fluorescent cells. TPP1-siRNA cell lines were compared with FAM-marked negative control (siNC group) and blank control (mock group).

RNA extraction and quantitative real-time polymerase chain reaction (PCR)

Total cell RNA was extracted using Trizol reagent (Invitrogen, US). The concentration and quality of RNA were evaluated by Nanodrop 2000 Spectrophotometer (Thermo Fisher Scientific). Reverse transcribed cDNA was synthesized from $0.1 \mu \mathrm{g}-5 \mu \mathrm{g}$ of total RNA by the RecertAidTM first strand cDNA Synthesis Kit (Fermentas, Canada) at $42^{\circ} \mathrm{C}$ for $60 \mathrm{~min}$, followed by $70^{\circ} \mathrm{C}$ for $5 \mathrm{~min}$ and $4^{\circ} \mathrm{C}$ afterward. Real-time PCR was performed with SYBR Premix Ex TaqTM (Takara, Japan) in a $12.5 \mu$ reaction volume using the CFX ConnectTM Real-Time PCR detection system (Bio-Rad, US). Primers designed for GAPDH, hTERT, and TPP1 were shown in Table S2. All values were normalized to GAPDH and the 2- $\triangle \triangle \mathrm{Ct}$ method was used to estimate the fold change of gene expression over control samples.

Protein extraction and Western Blot

Cells were digested using $1 \mathrm{ml}$ Trypsin-EDTA Solution (Beyotime, China) and then decomposed by $500 \mu \mathrm{l}$ RIPA Lysis Buffer per 100 packed cell volume (PCV). Western blot was carried out to evaluate TPP1 and hTERT protein expression. Primary antibodies targeting TPP1 (ab57595, Abcam, USA, dilution of 1:750), hTERT (ab32020, Abcam, USA, dilution of 1:1000), and $\beta$-actin (Santa Cruz, US, dilution of 1:2000) were incubated at $4^{\circ} \mathrm{C}$ overnight. ECL (Advansta, USA) was used to visualize the specific bands. Auto 
radiographs were recorded onto X-ray films (Eastman Kodak Co, USA) and the ImageJ software was applied to analyze the signal intensity of bands.

Gene expression of TPP1 in publicly available databases

Eligible microarray dataset (GEO accession number: GSE7803) including gene expressions of normal cervical tissues, CINs and cervical cancer tissues was used to assess the expression of TPP1 in different cervical lesions. Test of ANOVA was applied. Oncomine gene expression array dataset was queried to analyze TPP1 mRNA expression in cervical cancer and normal tissues. The defined parameters used to filter datasets were $\mathrm{p}$-value $<1 \mathrm{E}-4$ (Student's t-test), fold-change $>2$, and genes ranking in the top $10 \%$. One dataset of Zhai Cervix met the criteria and was selected to assess expression of TPP1 in cervical cancer tissues and normal tissues.

\section{Statistical analysis}

The association of TPP1 expression and clinical-pathological characteristics was assessed using the Chisquare test. Kaplan-Meier curve with a log-rank test was performed to compare overall survival of cancer patients by different expressions of TPP1 or hTERT. To test the prognosis value of TPP1 high expression or TPP1/hTERT high co-expression, multivariate analysis using the Cox proportional hazards model was performed, adjusted for other well-known prognosis factors, i.e., age, tumor diameter, FIGO stage, differentiation, lymphatic invasion, vagina invasion, parauterus invasion, pathological subtype. Hazard ratio (HR) with a 95\% confidence interval $(\mathrm{Cl})$ was estimated. The mutual relationship of TPP1 and hTERT expression in different cervical lesions was assessed by bivariate correlation analysis (Spearman coefficients). To assess the association between TPP1 high expression and different cervical lesions, odds ratio with $95 \% \mathrm{Cl}$ was estimated using binary logistic regression. Results of in vitro analysis were given as mean \pm standard error of the mean (SEM) with a minimum of three repetitions and a student's ttest was conducted. Statistical analyses were performed in SPSS 26 (SPSS Inc., Cary, NC, USA). All statistical analyses were two-sided and a $\mathrm{P}$ value of $<0.05$ was considered statistically significant.

\section{Results}

\section{Expression of TPP1 in normal cervix, CINs and cervical cancers}

Figure 1A and 1B showed protein expression of TPP1 in different cervical tissues of 274 participants, including 16 normal cervical samples, 102 CINs lesions and 156 samples of cervical cancers patients. There was $6.3 \%$ (1/16) of participants with normal cervixes showed TPP1 high expression. However, the percentage of patients with high TPP1 expression increased in parallel with the augment in CIN grade (9.6\% [5/52] in CIN-I, 31.8\% [7/22] in CIN-II and 67.9\% [19/28] in CIN-III) and reached 77.0\% (107/139) in cervical squamous cell carcinoma (SCC) patients and 82.4\% (14/17) in cervical adenocarcinoma (AC)/ adenosquamous carcinoma (ASC) patients. Compared with normal cervix, both CIN-III $(P=0.002)$ and 
cervical cancer (SCC or AC/ASC patients, $\mathrm{P}<0.001)$ showed a significantly higher odds ratio of TPP1 high expression (Fig. 1B).

In concert with high TPP1 protein expression detected in cervical cancer samples, higher expression of the TPP1 gene was validated in external and publicly available databases (GSE7803 and Oncomine). In the dataset of GSE7803, 10 normal cervical tissues, $7 \mathrm{CINs}$, and 21 cervical cancers were identified. When compared with normal tissues, TPP1 was higher expressed in $\mathrm{CINs}(\mathrm{P}<0.05)$ and highest expressed in cervical carcinoma $(P<0.001$, Fig. 1C). Similarly, in the dataset of Zhai Cervix from Oncomine including 10 normal cervical tissues and 21 cervical cancer cases, TPP1 gene was significantly overexpressed in cervical cancers compared with normal cervical tissues in $(P<0.001$, Fig. 1D). These results suggested that high TPP1 expression was associated with cervical cancer development and disease progression.

\section{Association of TPP1 expression with clinical-pathological factors and overall survival of cervical cancer patients}

Table 1 presented the association between the expression of TPP1 protein and clinical-pathological factors of cervical cancer patients. Expression level of TPP1 was related to pathological differentiation ( $P$ $=0.005)$, lymphatic invasion $(P=0.047)$, and vagina invasion $(P=0.022)$. Whereas, it was not related to patient's age $(P=0.643)$, tumor diameter $(P=0.376)$, FIGO stage $(P=0.990)$, parauterus invasion $(P=$ $0.480)$ or pathological subtypes $(P=0.616)$. 
Table 1Association of TPP1 expression and clinicopathological characteristics of cervical cancer

\section{Characteristics}

Age (years)

$\leq 45$

$\otimes 45$

Tumor diameter $(\mathrm{cm})$

$\leq 4$

$\llbracket 4$

\section{FIGO stage}

I

II

Differentiation

Poorly

Moderately

Well

Lymphatic invasion

Negtive

Positive

Vagina invasion

Invasion

No invasion

Parauterus invasion

Invasion

No invasion

Pathologic type

SCC

AC/ASC

Abbreviations: AC: adenocarcinoma; ASC: adenosquamous carcinoma; FIGO: the International Federation of Gynecology and Obstetrics; hTERT: human telomerase reverse transcriptase; SCC: squamous cell carcinoma; TPP1: telomere-binding protein 1.
Low expression $\quad P$ value

0.643

19

16

0.376

24

11

0.990

15

20

0.006

8

19

8

0.047

27

8

0.022

43

20

15

0.480

34

12

87

23

0.616

32

3 
Page 8/22 
Table 2Multivariable-adjusted hazard ratio of overall mortality among cervical cancer patients

\begin{tabular}{|c|c|c|c|c|c|c|}
\hline & \multirow{2}{*}{$\begin{array}{l}\text { No. } \\
\text { death }\end{array}$} & \multirow{2}{*}{$\begin{array}{l}\text { No. } \\
\text { patients }\end{array}$} & \multicolumn{2}{|c|}{ Cox regression model 1} & \multicolumn{2}{|c|}{ Cox regression model 2} \\
\hline & & & $\begin{array}{l}\text { Hazard ratio } \\
(95 \% \mathrm{Cl})\end{array}$ & $\begin{array}{l}P \\
\text { value }\end{array}$ & $\begin{array}{l}\text { Hazard ratio } \\
(95 \% \mathrm{Cl})\end{array}$ & $\begin{array}{l}P \\
\text { value }\end{array}$ \\
\hline Age (years) & 65 & 156 & $1.03(1.00-1.06)$ & 0.024 & $1.03(1.00-1.06)$ & 0.038 \\
\hline $\begin{array}{l}\text { Tumor diameter } \\
\text { (cm) }\end{array}$ & 65 & 156 & $1.14(0.95-1.37)$ & 0.173 & $1.15(0.96-1.38)$ & 0.140 \\
\hline
\end{tabular}

FIGO stage

\begin{tabular}{lllllll} 
I & 17 & 67 & Reference & \multicolumn{2}{l}{ Reference } \\
\hline II & 48 & 89 & $2.44(1.23-4.86)$ & 0.011 & $2.34(1.17-4.67)$ & 0.016
\end{tabular}

\section{Differentiation}

$\begin{array}{lllllll}\text { Poor } & 30 & 68 & \text { Reference } & 0.192 & \text { Reference } & 0.169 \\ \text { Moderate } & 26 & 70 & 0.72(0.41-1.26) & 0.68(0.39-1.18) \\ \text { Well } & 9 & 18 & 1.47(0.66-3.23) & 1.36(0.62-2.97)\end{array}$

\section{Lymphatic invasion}

\begin{tabular}{lllllll} 
Negative & 35 & 98 & Reference & \multicolumn{3}{l}{ Reference } \\
Positive & 30 & 58 & $1.34(0.78-2.29)$ & 0.291 & $1.29(0.75-2.22)$ & 0.354
\end{tabular}

\section{Vagina invasion}

\begin{tabular}{lllllll} 
Negative & 33 & 93 & Reference & \multicolumn{3}{l}{ Reference } \\
Positive & 32 & 63 & $1.19(0.65-2.19)$ & 0.566 & $1.15(0.63-2.08)$ & 0.653
\end{tabular}

\section{Parauterus invasion}

\begin{tabular}{lllllll} 
Negative & 42 & 110 & Reference & \multicolumn{3}{l}{ Reference } \\
Positive & 23 & 46 & $1.03(0.60-1.77)$ & 0.908 & $1.03(0.60-1.77)$ & 0.910
\end{tabular}

\section{Pathological type}

\begin{tabular}{lllllll} 
SCC & 57 & 139 & Reference & \multicolumn{3}{l}{ Reference } \\
AC/ASC & 8 & 17 & $0.93(0.42-2.06)$ & 0.860 & $0.88(0.40-1.94)$ & 0.747
\end{tabular}




\section{TPP1expression}

$\begin{array}{lllllll}\text { Low expression } & 10 & 35 & \text { Reference } & & \text { NA } \\ \text { High expression } & 55 & 121 & 2.03(0.99-4.16) & 0.054 & \text { NA } & \text { NA }\end{array}$

\section{TPP1/hTERT}

coexpression

\begin{tabular}{lcccccc}
$\begin{array}{l}\text { Without co-high } \\
\text { expression }\end{array}$ & 15 & 55 & NA & NA & Reference \\
\\
$\begin{array}{l}\text { With co-high } \\
\text { expression }\end{array}$ & 50 & 101 & NA & & $2.01(1.10-3.67)$ & 0.024 \\
\hline
\end{tabular}

Abbreviations: AC: adenocarcinoma; ASC: adenosquamous carcinoma; $\mathrm{Cl}$ : confidence interval; FIGO: the International Federation of Gynecology and Obstetrics; hTERT: human telomerase reverse transcriptase; NA: not applicable; SCC: squamous cell carcinoma; TPP1: telomere-binding protein 1. 
Table 3 Correlation of expression of TPP1 and hTERT in cervical tissues

\begin{tabular}{|c|c|c|c|c|c|c|}
\hline $\begin{array}{l}\text { Tissue } \\
\text { type }\end{array}$ & $\begin{array}{l}\text { TPP1 } \\
\text { high, } \mathrm{n}\end{array}$ & $\begin{array}{l}\text { TPP1 } \\
\text { low, n }\end{array}$ & $\begin{array}{l}\text { Concordance } \\
\text { (\%) }\end{array}$ & $\begin{array}{l}\text { Spearman } \\
\text { coefficient(r) }\end{array}$ & correlation & $P$ value \\
\hline
\end{tabular}

\section{Normal tissue}

hTERT high 113

81.3

0.569

0.022

hTERT low $\quad 0 \quad 12$

\section{CIN I}

$\begin{array}{llllll}\text { hTERT high } & 3 & 25 & 48.1 & 0.322 & 0.020 \\ & & & & \end{array}$

\section{CIN II}

hTERT high $4 \quad 4$

68.2

0.496

0.019

hTERT low $\quad 3 \quad 11$

\section{CIN III}

hTERT high 13

75.0

0.648

$<0.001$

hTERT low $\quad 6 \quad 8$

SCC

hTERT high $\quad 87 \quad 19$

hTERT low $\quad 20 \quad 13$

AC/ASC

hTERT high $\quad 14 \quad 1$

94.1

0.487

0.047

hTERT low $\quad 0 \quad 2$

All

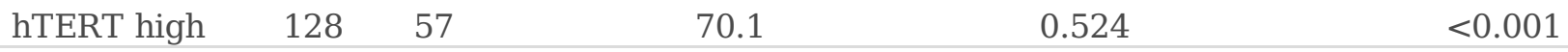

hTERT low $\quad 25 \quad 64$

Abbreviations: AC: adenocarcinoma; ASC: adenosquamous carcinoma; CIN: intraepithelial neoplasia; hTERT: human telomerase reverse transcriptase; SCC: squamous cell carcinoma; TPP1: telomere-binding protein 1. 
The prognostic value of TPP1 expression in overall survival was further evaluated using Kaplan-Meier analysis and multivariable COX regression. Among 156 cervical cancer patients, 65 (41.7\%) of them died during the follow-up. The median overall survival was 108 months and the five-year survival rate was 68.8\%. Compared with TPP1 lowly expressed cervical cancer patients, a significantly worse survivorship was found in their counterparts with high expression of TPP1 $\left(P_{\text {log-rank }}=0.047\right.$, Fig. 2A). Multivariable analysis showed that high expression of TPP1 was marginally associated with a poor prognosis of cervical cancer $(\mathrm{HR}=2.03$ [95\% Cl 0.99-4.16], Table 2). Kaplan-Meier analysis showed that higher expression of hTERT trended to be associated with worse but non-significant survival $\left(P_{\text {log-rank }}=0.060\right.$, Fig. S1B). 64.7\% (101/156) of cancer patients had high coexpression of both TPP1 and hTERT (Table 2). Patients with high coexpression of TPP1/hTERT showed significantly worse overall survival $\left(P_{\text {log-rank }}=0.005\right.$, Fig. 2B), carrying $2.01(95 \% \mathrm{Cl} 1.10-3.67)$ times higher mortality risk than those without high coexpression (Table 2). These data indicated that TPP1 and hTERT was associated with patient survival in cervical cancers.

\section{Correlation between TPP1 and hTERT expressions in cervical lesions}

Association between TPP1 and hTERT expression was further tested in different cervical lesions, both of which were measured in the same batch of samples $(n=274)$. Similar to TPP1, the percentage of patients with high expression of hTERT increased with CINs (57.7\% [30/52] in CIN-I, 59.1\% [13/22] in CIN-II and $60.7 \%$ [17/28] in CIN-III) and reached 76.3\% (106/139) in SCC and 88.2\% (15/17) in AC/ASC (Table 3). Spearman correlation analysis revealed positive correlations between TPP1 and hTERT expression both in the overall levels $(r=0.524, P<0.001)$ and in participants with different cervical lesions (Table 3$)$. These findings demonstrated an inter-regulation between TPP1 and hTERT that might determine cervical cancer development.

\section{TPP1 regulated the expression of hTERT in cervical cancer cells}

To further explore the regulatory mechanism of hTERT by TPP1, we transfected TPP1-siRNA into three human cervical cancer cell lines, i.e., Hela, Siha, and C33a cells. Fluorescence microscope, real-time PCR, and western blot were used to confirm transfection efficiency and depletion of TPP1 (Fig. 3A, B and D). The transfecting efficiencies in Hela, Siha, and C33a cells were $95 \%, 90 \%$, and $85 \%$, respectively (Fig. 3A). Compared with control cells, TPP1 expression in three cell lines was significantly reduced in the TPP1siRNA transfected cells, both at mRNA (Fig. 3B) and protein levels (Fig. 3D), suggesting a satisfied knockdown effect of TPP1. We found that hTERT protein expression was downregulated in TPP1 depleted cells at both the mRNA (Fig. 3C) and protein levels (Fig. 3E) in all cell lines. Compared with their 
mock controls, $h T E R T$ mRNA expressions in Hela, Siha, and C33a cells reduced by $38.5 \%, 81.7 \%$, and $51.5 \%$, respectively (Fig. 3C). And the deducted expressions of the hTERT protein were $70.7 \%, 57.0 \%$, and $85.2 \%$, respectively (Fig. 3E). These results suggested that TPP1 regulated $h T E R T$ expression in cervical cancer cells.

\section{Discussion}

A substantial increase of cervical cancer was seen in developing countries, especially among young females [20], making it urgent to understand the driving reason for cancer development and to find new biomarkers for early detection of this disease and potential therapeutic targets. This study indicated that high TPP1 expression was significantly related to CIN-III and cervical cancers. High TPP1 expression was a strong risk factor of poor survival for cervical cancer patients, especially when co-highly expressed with hTERT. TPP1 expression positively correlated with the expression of hTERT in cervical cancer and depletion of TPP1 substantially decreased hTERT expression. These findings indicated that TPP1 might be both a clinical biomarker for early malignant tumors and a prognostic factor in cervical cancer, and it might also be served as a therapeutic target by regulating hTERT expression.

Previous studies found that higher expression of TPP1 was involved in telomere elongation and contributed to the increase of malignant potential in the pathogenesis of B-cell leukemia and colorectal cancer [21-23]. Huang et al. also found that TPP1 was overexpressed in gastric cancer compared to adjacent normal tissue, and further confirmed that TPP1 promoted cancer cell proliferation [24]. We found that TPP1 was related to the progress of cervical cancer malignancy, as shown by continuously increasing positive expression rates from CIN-I, CIN-II, CIN-III, to SCC and AC/ASC. Ki-67 encoded by the MKI67 gene is a marker of cellular proliferation closely related to tumor malignancy. We also assessed the correlation between TPP1 and MKI67 gene expressions using TCGA data from GEPIA database in cervical cancer. Interestingly, we found mRNA expression of TPP1 was positively correlated with the expression of MKI67 (Fig. S2), suggesting that TPP1 could be linked to proliferation and malignancy of cervical cancer. In addition, our results suggested that increased TPP1 expression was an early event during epithelial malignant transformation and cervical cancer initiation, and a substantial-high expression rate started in $\mathrm{CIN}$-III. The result was verified by a small external analysis when we analyzed the expression of TPP1 in the dataset of GSE7803. Our study suggested that TPP1 might be a potential predictive biomarker for early cervical cancers.

TPP1 is responsible for recruiting telomerase to the telomere and maintaining telomerase processivity with the inseparable participation of hTERT [25]. But the interaction of TPP1 and hTERT in cervical cancer was still unclear. Correlative analysis of clinical samples in the current study revealed that TPP1 and hTERT expressions were positively correlated in cervical cancer. TPP1 might regulate hTERT expression as it was crucial for hTERT recruitment to telomeres and telomere elongation [26]. In vitro results verified that the expression of hTERT was decreased at both mRNA and protein levels when inhibiting TPP1 expression. Although there lacks direct proof of hTERT expression by TPP1 for the moment, we speculated that the hTERT might be regulated by TPP1 through several pathways. One possible way 
could be that the TEL patch on the surface of TPP1 directly interacted with the TEN domains of hTERT to recruit telomerase to telomeres [27-29]. Knockdown of TPP1 could decrease the telomerase activity by affecting TPP1 and hTERT combination, which further reduced hTERT expression. Additionally, some studies reported that TPP1 bound to POT1 and interacted with POT1 to affect telomerase regulation at chromosome ends [30]. The C-terminal portion of human POT1 (POT1C) was vital for the POT1C-TPP1 interaction and disruption of the interaction was essential to prevent initiation of genome instability permissive for tumorigenesis [12]. HTERT activity could be partially activated by overexpression of TPP1POT1 via the insertion of fingers domain (IFD) at hTERT in a TPP1-dependent manner [31]. Both of these two pathways were critical for the functions of TPP1-Htert [25]. Moreover, c-Myc was involved in regulating the TPP1-hTERT complex via phosphatidylinositol 3-kinase/Akt, which regulated vascular smooth muscle cell cycle progression and cell proliferation [32]. Besides, other research demonstrated that TPP1 might be also involved in regulation of telomere shortening by interacting with hTERT as an upstream regulator in human cells [33]. It is possible that TPP1 expression is elevated earlier than the activation of hTERT and hTERT activation by the TPP1 signaling pathway might lead to the occurrence or progression of cervical cancer.

It was interesting to note that high TPP1 expression was a strong predictor of poor survival for cervical cancer and patients with high co-expression of TPP1 and hTERT exhibited a much worse survival than those without such high co-expression. TPP1 expression was related to tumor differentiation, local invasion and distant metastasis and its expression in AC/ASC was higher than that in SCC, indicating a positive correlation between TPP1 expression and the aggressiveness of cancer. High expression of TPP1 in tumor cells can protect telomere end structure from fusion and DNA damage, and thus enhance genomic stability and improve cellular immortalization potential [34]. Telomerase of tumor cells might be highly activated when both TPP1 and hTERT were overexpressed, which could further result in higher invasiveness and poorer prognosis. We also did an exploration analysis to the expression difference of TPP1 gene in other cancer types and their corresponding controls using Oncomine database. The results revealed that TPP1 was also overexpressed in several other cancers such as esophageal carcinoma, stomach adenocarcinoma and liver hepatocellular carcinoma, Increased expression of TPP1 was associated with worse survival in these cancers (Fig. S3). Overexpressed TPP1 might promote tumor malignancy in specific cancers, implying the possibility of TPP1 being a prognosis predictor of these TPP1-highly-expressed cancers.

The strengths of the study include rigorous clinical data collection, a long-term systematic and prospective follow-up, and exploration of potential molecular mechanisms concerning TPP1 and hTERT and extended bioinformatics analysis. In vitro experiments using three different well-known cervical cancer cell lines were well designed and each finding was validated through several methods, ensuring robustness of the results. However, several limitations warrant attention. Despite that we have enrolled all cervical cancer patients during the study period, it is a single hospital-based study with limited study sample size, which could hinder its generalizability. Therefore, further large prospective studies in different populations are warranted to evaluate the clinical application of TPP1. How TPP1 interacts with 
and regulates hTERT, and by which means TPP1 promotes the malignant phenotype of cervical cancer are under active investigation.

\section{Conclusions}

TPP1 expression increased significantly in late precursor lesions (CIN-III) and cervical cancers compared with normal cervical tissue. High expression of TPP1 indicated a worse survival in cervical cancer and cohigh expression with hTERT can better predict a worse survival. Our results also revealed that TPP1 and hTERT expression were positively correlated by both in vivo and in vitro analysis. High expression of TPP1 may be an early event during cervical cancer development and a prognosis factor, making it a potential predictor for early cancer detection and potential target for cervical cancer treatment. Meanwhile, TPP1 might influence tumor development via regulating hTERT expression, whereas detailed underlining pathways warrant further investigations.

\section{Abbreviations}

AC: adenocarcinoma; ASC:adenosquamous carcinoma; Cl:confidence interval; CIN:intraepithelial neoplasia; FIGO:International Federation of Gynecology and Obstetrics; hTERT:human telomerase reverse transcriptase; IHC:immunohistochemistry; OS:overall survival; PCR:polymerase chain reaction; POT1:protection of telomeres 1; SCC:squamous cell carcinoma; TPP1:telomere-binding protein 1.

\section{Declarations}

\section{Ethics approval and consent to participate}

This study was approved by the Institutional Ethical Review Board of the Zhongnan Hospital of Wuhan University (ethical approval number: 2014054).

\section{Consent for publication}

Not applicable.

\section{Availability of data and materials}

Data available from the authors upon reasonable request and with permission of Zhongnan Hospital of Wuhan University in China.

\section{Competing interests}

All authors confirm and declare that no conflicts of interest exist.

\section{Funding}


This research was funded by the National Natural Science Foundation of China (NO. 81472799) and Hubei Province Natural Science Foundation for Innovation Groups (NO. 2013CFA006).

\section{Authors' contributions}

Conceptualization, QL.W, FX.Z, and YF.Z; study design: QL.W, CF.G, and QJ.W; clinical data collection, QL.W and HY; wet lab work: QL.W; bioinformatic analysis: CF.G; visualization, QL.W, CF.G, and QJ.W; manuscript writing: CF.G and QL.W; revision: all authors; supervision, YF.Z, FX.Z, and QJ.W; funding: YF.Z. All authors have read and approved the final version of the manuscript.

\section{Acknowledgements}

We acknowledge all the involved participants for their agreement of using their clinical pathological data in this study.

\section{References}

1. Bray F, Ferlay J, Soerjomataram I, Siegel RL, Torre LA, Jemal A. Global cancer statistics 2018: Globocan estimates of incidence and mortality worldwide for 36 cancers in 185 countries. CA Cancer J Clin. 2018,68:394-424.

2. Forouzanfar MH, Foreman KJ, Delossantos AM, Lozano R, Lopez AD, Murray CJ, et al. Breast and cervical cancer in 187 countries between 1980 and 2010: A systematic analysis. Lancet. 2011,378:1461-84.

3. Shay JW. Role of telomeres and telomerase in aging and cancer. Cancer Discov. 2016,6:584-93.

4. de Lange T. Shelterin: The protein complex that shapes and safeguards human telomeres. Genes Dev. 2005,19:2100-10.

5. Sexton AN, Regalado SG, Lai CS, Cost GJ, O'Neil CM, Unnov FD, et al. Genetic and molecular identification of three human tpp1 functions in telomerase action: Recruitment, activation, and homeostasis set point regulation. Genes Dev. 2014,28:1885-99.

6. Wang F, Podell ER, Zaug AJ, Yang Y, Baciu P, Cech TR, et al. The pot1-tpp1 telomere complex is a telomerase processivity factor. Nature. 2007,445:506-10.

7. Xin H, Liu D, Wan M, Safari A, Kim H, Sun W, et al. Tpp1 is a homologue of ciliate tebp-beta and interacts with pot1 to recruit telomerase. Nature. 2007,445:559-62.

8. Chen C, Gu P, Wu J, Chen X, Niu S, Sun H, et al. Structural insights into pot1-tpp1 interaction and pot1 c-terminal mutations in human cancer. Nat Commun. 2017,8:14929.

9. Rao T, Lubin JW, Armstrong GS, Tucey TM, Lundblad V, Wuttke DS. Structure of est3 reveals a bimodal surface with differential roles in telomere replication. Proc Natl Acad Sci U S A. 2014,111:214-8.

10. Rice C, Shastrula PK, Kossenkov AV, Hills R, Baird DM, Showe LC, et al. Structural and functional analysis of the human pot1-tpp1 telomeric complex. Nat Commun. 2017,8:14928. 
11. Dogan F, Biray Avci C. Correlation between telomerase and mtor pathway in cancer stem cells. Gene. 2018,641:235-9.

12. Kim H, Yoo JE, Cho JY, Oh BK, Yoon YS, Han HS, et al. Telomere length, tert and shelterin complex proteins in hepatocellular carcinomas expressing "stemness"-related markers. J Hepatol. 2013,59:746-52.

13. Lipinska N, Romaniuk A, Paszel-Jaworska A, Toton E, Kopczynski P, Rubis B. Telomerase and drug resistance in cancer. Cell Mol Life Sci. 2017,74:4121-32.

14. Yan T, Ooi WF, Qamra A, Cheung A, Ma D, Sundaram GM, et al. Hoxc5 and mir-615-3p target newly evolved genomic regions to repress htert and inhibit tumorigenesis. Nat Commun. 2018,9:100.

15. Bisht K, Smith EM, Tesmer VM, Nandakumar J. Structural and functional consequences of a disease mutation in the telomere protein tpp1. Proc Natl Acad Sci U S A. 2016,113:13021-6.

16. Trybek T, Kowalik A, Góźdź S, Kowalska A. Telomeres and telomerase in oncogenesis. Oncol Lett. 2020,20:1015-27.

17. Ivancich M, Schrank Z, Wojdyla L, Leviskas B, Kuckovic A, Sanjali A, et al. Treating cancer by targeting telomeres and telomerase.Antioxidants (Basel). 2017,6:15.

18. Jafri MA, Ansari SA, Alqahtani MH, Shay JW. Roles of telomeres and telomerase in cancer, and advances in telomerase-targeted therapies. Genome Med. 2016,8:69.

19. Li J, Cao X, Fang Y, Liao ZE, Liu YY, Huang BD, et al. Overexpression of htert in potentially malignant colorectal laterally spreading tumors. Mol Med Rep. 2013,7:1409-12.

20. Chen W, Zheng R, Baade PD, Zhang S, Zeng H, Bray F, et al. Cancer statistics in china, 2015.CA Cancer J Clin. 2016,66:115-32.

21. Poncet D, Belleville A, t'kint de Roodenbeke C, Roborel de Climens A, Ben Simon E, Merle-Beral H, et al. Changes in the expression of telomere maintenance genes suggest global telomere dysfunction in bchronic lymphocytic leukemia. Blood. 2008,111:2388-91.

22. Kojima K, Hiyama E, Otani K, Ohtaki M, Fukuba I, Fukuda E, et al. Telomerase activation without shortening of telomeric 3 '-overhang is a poor prognostic factor in human colorectal cancer. Cancer Sci. 2011,102:330-5.

23. Grill S, Bisht K, Tesmer VM, Shami AN, Hammoud SS, Nandakumar J. Two separation-of-function isoforms of human tpp1 dictate telomerase regulation in somatic and germ cells. Cell Rep. 2019,27:3511-21.

24. Huang K, Chen S, Xie R, Jiang P, Yu C, Fang J, et al. Identification of three predictors of gastric cancer progression and prognosis. FEBS Open Bio. 2020,10:1891-9.

25. Tesmer VM, Smith EM, Danciu O, Padmanaban S, Nandakumar J. Combining conservation and species-specific differences to determine how human telomerase binds telomeres. Proc Natl Acad Sci U S A. 2019,116:26505-15.

26. Tejera AM, Stagno d'Alcontres M, Thanasoula M, Marion RM, Martinez P, Liao C, et al. Tpp1 is required for tert recruitment, telomere elongation during nuclear reprogramming, and normal skin 
development in mice. Dev Cell. 2010,18:775-89.

27. Dalby AB, Hofr C, Cech TR. Contributions of the tel-patch amino acid cluster on tpp 1 to telomeric DNA synthesis by human telomerase. J Mol Biol. 2015,427:1291-303.

28. Nandakumar J, Bell CF, Weidenfeld I, Zaug AJ, Leinwand LA, Cech TR. The tel patch of telomere protein tpp1 mediates telomerase recruitment and processivity. Nature. 2012,492:285-9.

29. Zhong FL, Batista LF, Freund A, Pech MF, Venteicher AS, Artandi SE. Tpp1 ob-fold domain controls telomere maintenance by recruiting telomerase to chromosome ends. Cell. 2012,150:481-94.

30. Miyoshi T, Kanoh J, Saito M, Ishikawa F. Fission yeast pot1-tpp1 protects telomeres and regulates telomere length. Science. 2008,320:1341-4.

31. Chu TW, D'Souza Y, Autexier C. The insertion in fingers domain in human telomerase can mediate enzyme processivity and telomerase recruitment to telomeres in a tpp1-dependent manner. Mol Cell Biol. 2016,36:210-22.

32. Wang WL, Yeh YT, Chen LJ, Chiu JJ. Regulation of fibrillar collagen-mediated smooth muscle cell proliferation in response to chemical stimuli by telomere reverse transcriptase through cmyc.Biomaterials. 2014,35:3829-39.

33. Zheng YL, Zhang F, Sun B, Du J, Sun C, Yuan J, et al. Telomerase enzymatic component htert shortens long telomeres in human cells. Cell Cycle. 2014,13:1765-76.

34. Bernal A, Tusell L. Telomeres: Implications for cancer development. Int J Mol Sci. 2018,19: 294.

\section{Figures}




\section{A}
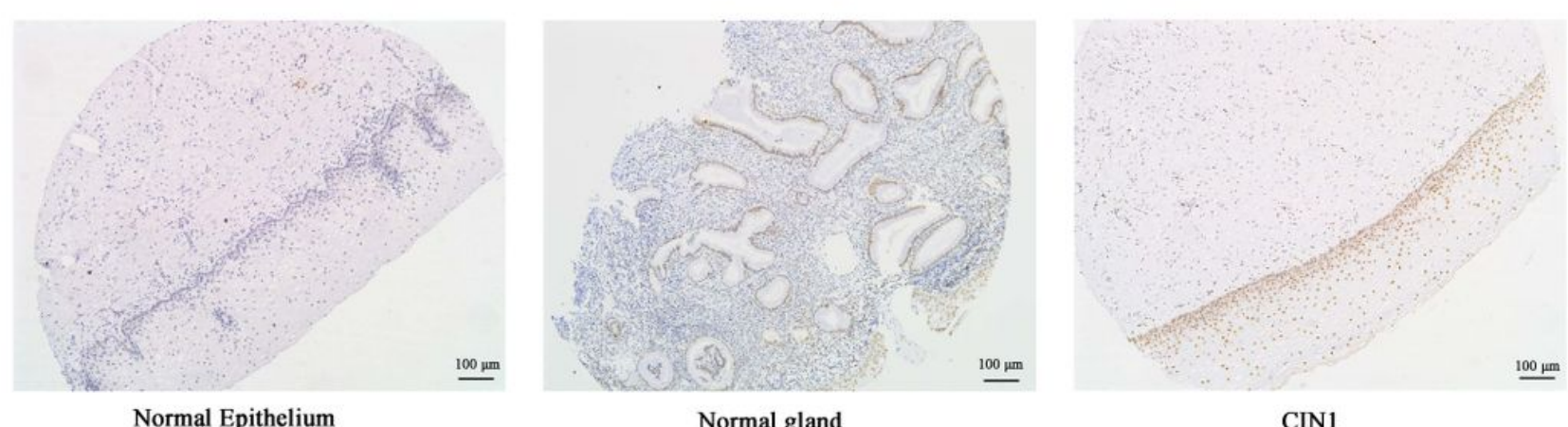

Normal gland

CIN1
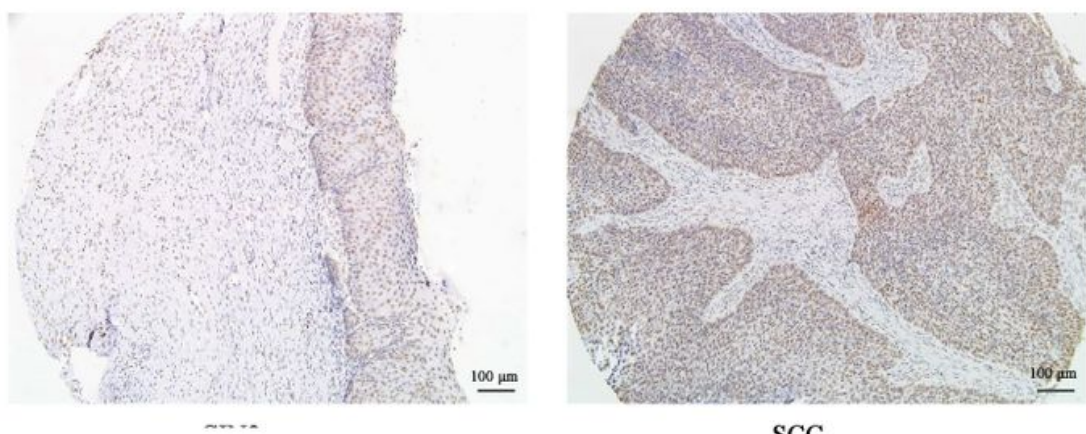

SCC

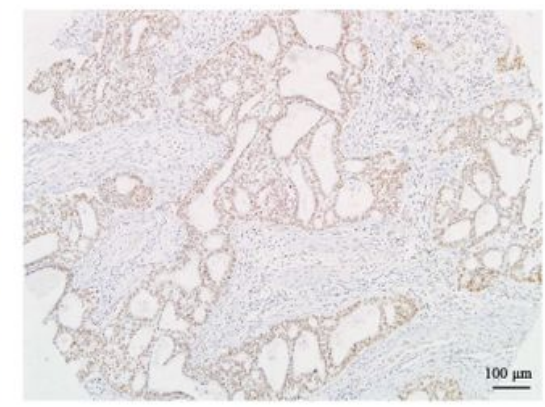

AC/ASC

B

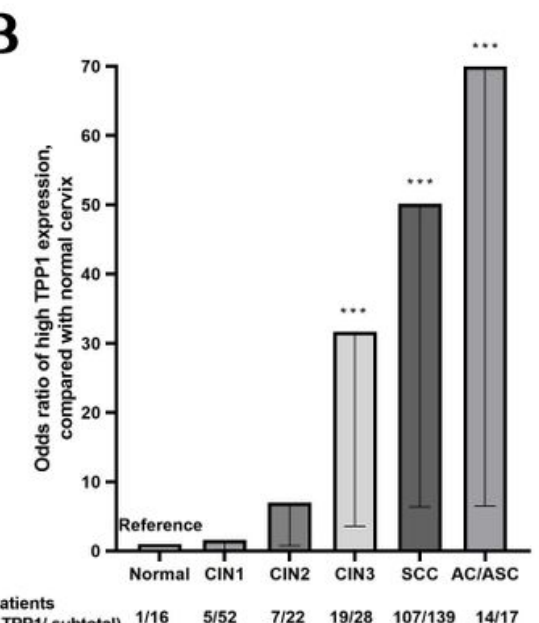

$\mathrm{C}$

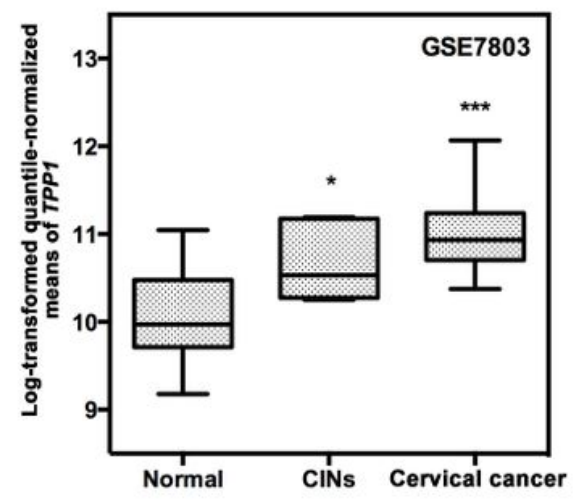

\section{D}

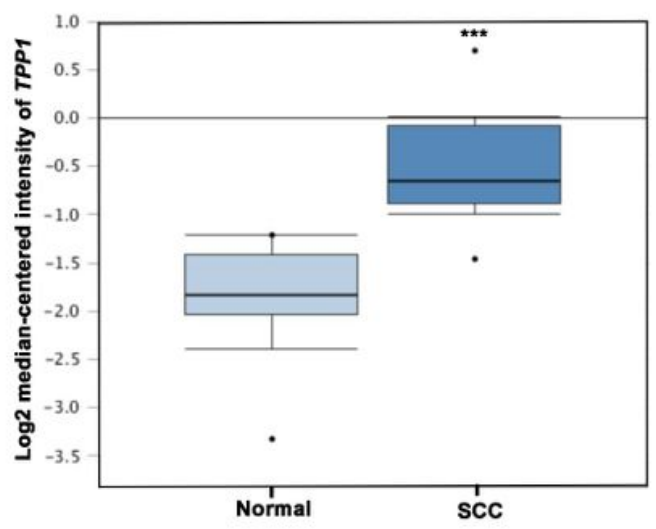

\section{Figure 1}

Expression of TPP1 in different cervical lesions. (A)Representative results of TPP1 expressions in normal, $\mathrm{CIN}-\mathrm{I}, \mathrm{CIN}-\mathrm{III}$ and cervical carcinoma tissues by immunohistochemistry ( $\mathrm{n}=274$, scale: $100 \mu \mathrm{m})$. (B)Odds ratio of TPP1 protein high expression amongCINs and cervical carcinoma tissues, whencompared with normal cervix $(n=274)$. (C)Gene expression of TPP1 in the dataset of GSE7803, comparing CIN and cervicalcancerwith normal cervix.(D) Gene expression of TPP1 in the dataset of Zhai Cervix from Oncomine, comparing cervical squamous cell carcinoma with normal cervix squamous epithelium.Abbreviations:AC: adenocarcinoma; ASC: adenosquamous carcinoma; CINs: intraepithelial neoplasia; SCC: squamous cell carcinoma; ${ }^{*} \mathrm{P}<0.05, * \star * \mathrm{P}<0.001$. 
A

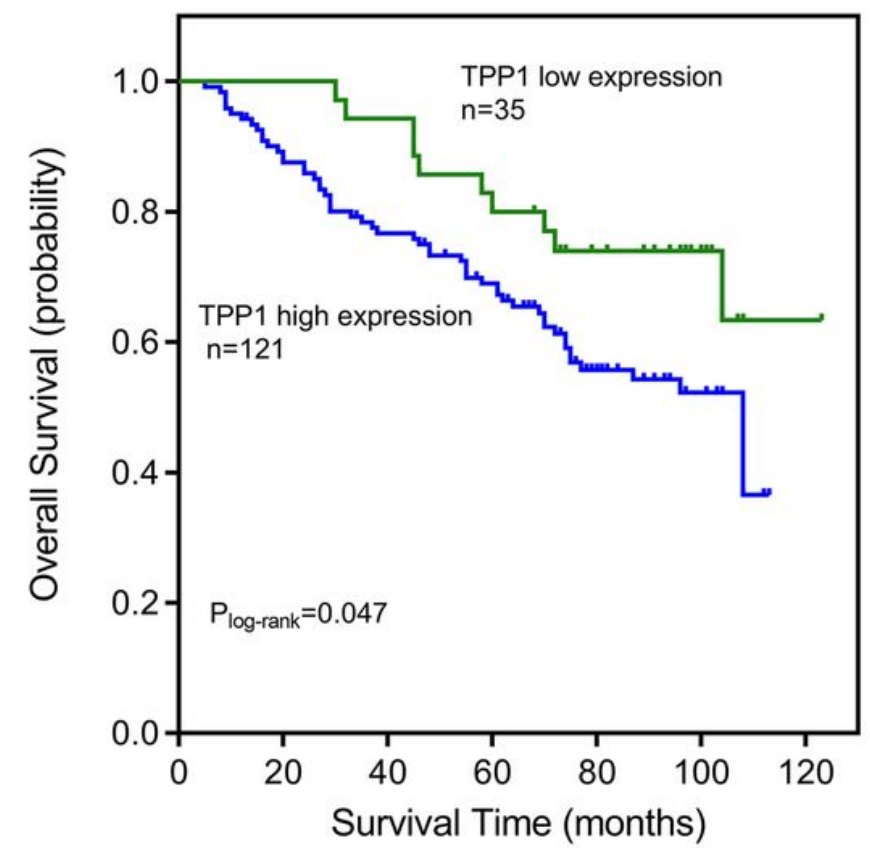

B

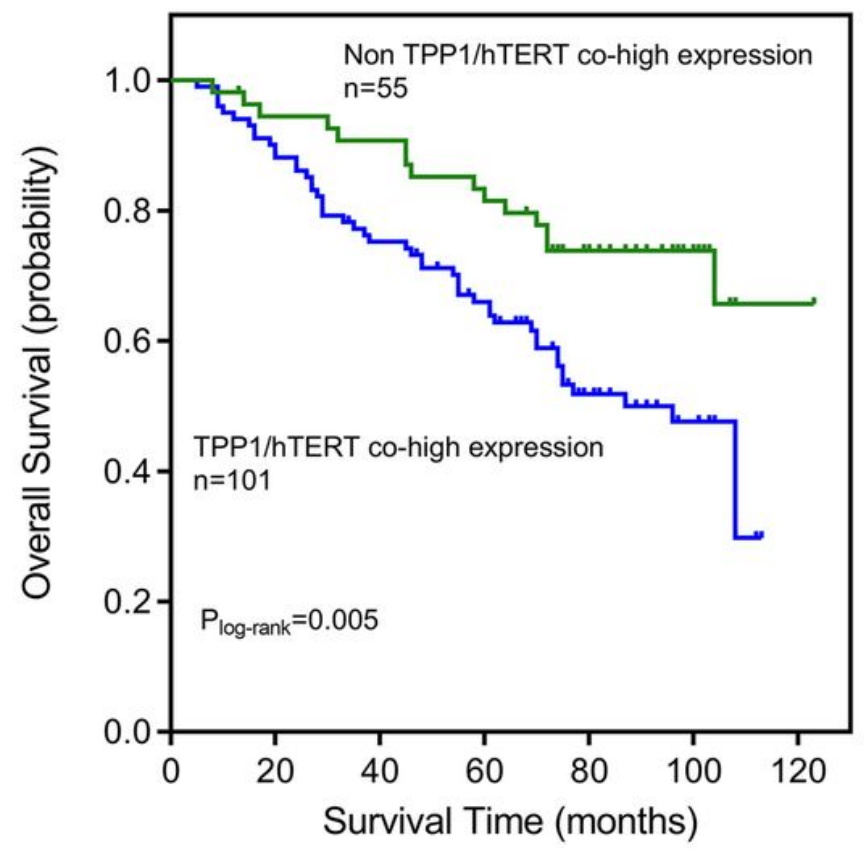

\section{Figure 2}

Kaplan-Meier curve of overall survival among cervical cancer patients by expression levels of TPP1 or together with hTERT. (A) High expression of TPP1 was associated with worse overall survival in cervical cancer patients $(n=156)$.(B) Patients with both TPP1 and hTERT high expression had worse overall survival than those without TPP1 and hTERT co-high expression $(n=156)$. 
A
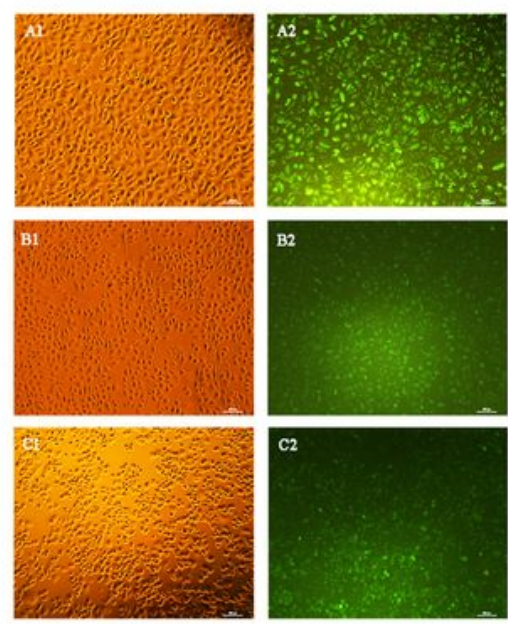

B

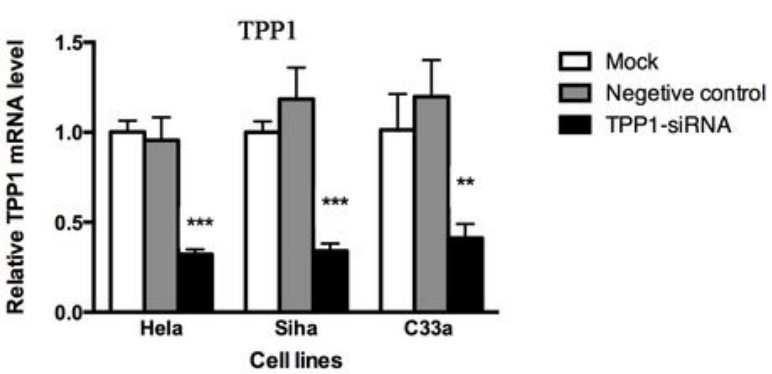

C

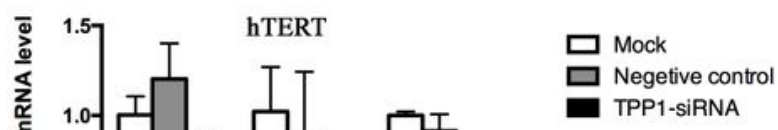

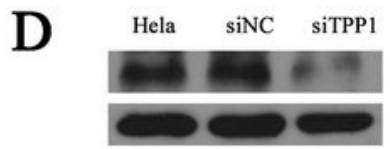

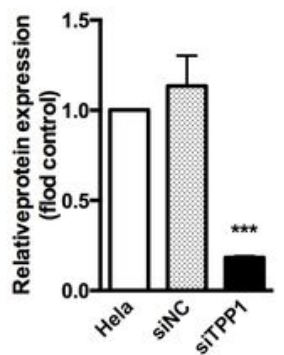

$\mathrm{E}$
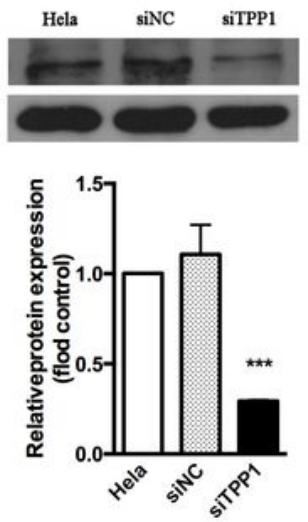
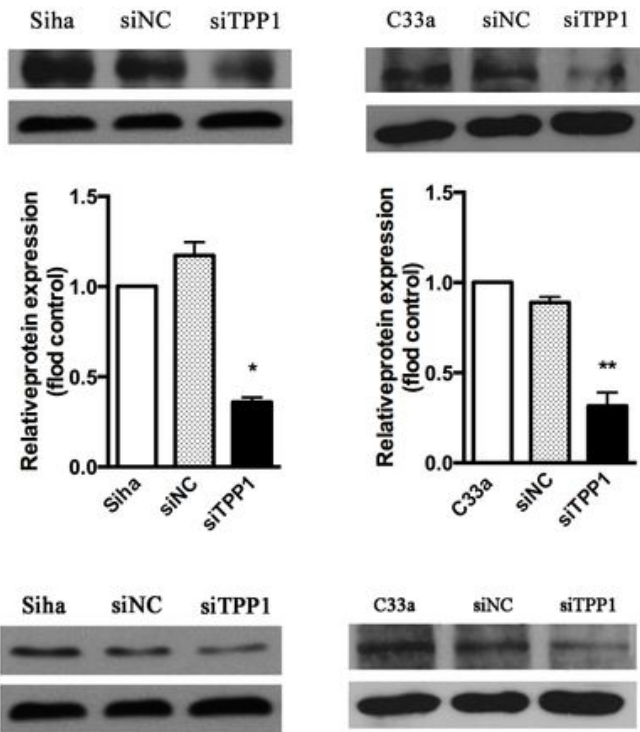

hTERT

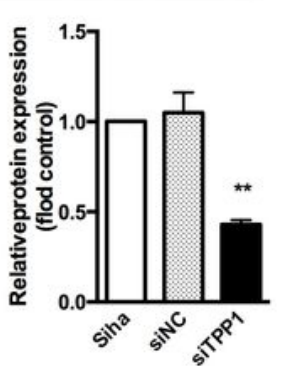

\section{Figure 3}

Knockdown of TPP1 regulated the expression of hTERT in three cell lines of cervical cancer. (A) Fluorescence microscopy was used to verify the knockdown effectiveness on cervical cancer cell lines of

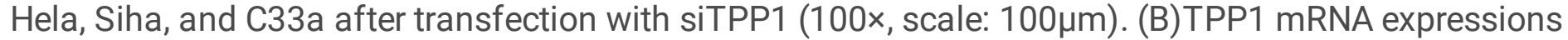
was decreased detected by real-time PCRafter siTPP1 transfection. (C) hTERT mRNA expression was downregulated after TPP1 knockdown detected by real-time PCR. (D)Decreaseof TPP1 protein expression 
detected by western-blot after siTPP1 transfection. (E) hTERT protein expression assessed by westernblot was down-regulated after TPP1 knockdown. Abbreviations: NC: negative control; * $p<0.05,{ }^{\star *} p<0.01$, $\star \star \star ~ p<0.001, n=3$.

\section{Supplementary Files}

This is a list of supplementary files associated with this preprint. Click to download.

- Additionalfile1SupplementaryMaterialsforBR.docx 